\title{
OPEN Assessing cerebral blood flow, oxygenation and cytochrome $c$ oxidase stability in preterm infants during the first 3 days after birth
}

\author{
Ajay Rajaram ${ }^{1,2}$, Daniel Milej ${ }^{1,2}$, Marianne Suwalski ${ }^{1,2}$, Lilian Kebaya ${ }^{3}$, Matthew Kewin ${ }^{1,2}$,
} Lawrence Yip ${ }^{1,2}$, Sandrine de Ribaupierre ${ }^{2,3}$, Victor $\mathrm{Han}^{3}$, Mamadou Diop ${ }^{1,2}$, Soume Bhattacharya ${ }^{3}$ \& Keith St. Lawrence ${ }^{1,2 \bowtie}$

A major concern with preterm birth is the risk of neurodevelopmental disability. Poor cerebral circulation leading to periods of hypoxia is believed to play a significant role in the etiology of preterm brain injury, with the first three days of life considered the period when the brain is most vulnerable. This study focused on monitoring cerebral perfusion and metabolism during the first $72 \mathrm{~h}$ after birth in preterm infants weighing less than $1500 \mathrm{~g}$. Brain monitoring was performed by combining hyperspectral near-infrared spectroscopy to assess oxygen saturation and the oxidation state of cytochrome coxidase (oxCCO), with diffuse correlation spectroscopy to monitor cerebral blood flow (CBF). In seven of eight patients, oxCCO remained independent of $C B F$, indicating adequate oxygen delivery despite any fluctuations in cerebral hemodynamics. In the remaining infant, a significant correlation between $\mathrm{CBF}$ and oxCCO was found during the monitoring periods on days 1 and 3 . This infant also had the lowest baseline CBF, suggesting the impact of CBF instabilities on metabolism depends on the level of blood supply to the brain. In summary, this study demonstrated for the first time how continuous perfusion and metabolic monitoring can be achieved, opening the possibility to investigate if $\mathrm{CBF} / \mathrm{oxCCO}$ monitoring could help identify preterm infants at risk of brain injury.

Premature birth is defined as a gestational age (GA) less than 37 weeks and has shown to strongly correlate with the development of adverse neurological outcomes such as cognitive and behavioural deficits and more severe disorders such as cerebral palsy ${ }^{1-3}$. The duration of gestation and an infant's weight at birth are factors that influence the likelihood of adverse effects. One of the most common brain injuries associated withpreterm birth is intraventricular hemorrhaging (IVH), which is characterized by bleeding in the germinal matrix and surrounding white matter. IVH has been found to occur in $20-25 \%$ of preterm infants born with very low birth weights (VLBW, $<1500 \mathrm{~g}$ ) and typically occurs within the first $72 \mathrm{~h}$ after birth ${ }^{4}$. Diagnosis is performed using cranial ultrasound (cUS) to visualize and grade cerebral hemorrhages. In most centers, standard practice calls for imaging within the first week of life and again within the first month ${ }^{5}$. A drawback with cUS is that it is not a prognostic technique as it only detects damage that has already occurred.

Although the pathogenesis of IVH is multifactorial, unstable cerebral blood flow (CBF), leading to periods of ischemia, is considered a contributing factor due to the confluence of a number of factors ${ }^{6}$ : an underdeveloped cerebrovascular system ${ }^{7}$, the absence of adequate cerebral autoregulation (i.e., the ability to maintain constant CBF during changes in blood pressure $)^{8}$, and little tolerance to flow reductions given the already very low basal $\mathrm{CBF}^{9}$. Studies have shown the potential of near-infrared spectroscopy (NIRS) monitoring of tissue saturation $\left(\mathrm{StO}_{2}\right)$ as a means of detecting precursors of preterm brain injury ${ }^{10-12}$. Correlating $\mathrm{StO}_{2}$ to arterial blood pressure has demonstrated that a sizable fraction of preterm infants has impaired cerebral autoregulation ${ }^{8}$. The SafeBoosC Phase II randomized clinical trial found that maintaining $\mathrm{StO}_{2}$ above $55 \%$ reduced the burden of hypoxia ${ }^{13,14}$. However, a link between $\mathrm{StO}_{2}$ and improved neurodevelopmental outcome has yet to be established ${ }^{15}$, likely due

${ }^{1}$ Imaging Program, Lawson Health Research Institute, London, ON, Canada. ${ }^{2}$ Department of Medical Biophysics, Western University, London, Canada. ${ }^{3}$ Division of Neonatal-Perinatal Medicine, Department of Pediatrics, London Health Sciences Centre, London, ON N6A 3K7, Canada. ${ }^{\circledR}$ email: ajay.rajaram@childrens.harvard.edu; kstlaw@ lawsonimaging.ca 


\begin{tabular}{|c|c|}
\hline Gestational age at birth (weeks + days) & $28 \pm 2(24+5$ to $31+1)$ \\
\hline Birth weight $(\mathrm{g})$ & $1123 \pm 344$ \\
\hline Sex (n) & 5 Male; 3 Female \\
\hline Apgar score $5 \mathrm{~min}$ & $6.7 \pm 3.2(1$ to 9$)$ \\
\hline Apgar score $10 \mathrm{~min}$ & $8.3 \pm 1.0(7$ to 9$)$ \\
\hline Mode of delivery & Vaginal (3), Caesarian (5) \\
\hline Doses of antenatal steroids & 1 dose (1), 2 doses (7) \\
\hline Delayed cord clamping & Yes (5), No (0) \\
\hline Mode of ventilation & $\begin{array}{l}\text { 6: continuous positive airway pressure, } \\
\text { 2: invasive mechanical ventilation }\end{array}$ \\
\hline Use of inotrope & Yes (1); No (7) \\
\hline IVH diagnosis (n, grade) & 1, Grade II right/Grade I left \\
\hline Left ventricular output (LVO, $\mathrm{ml} / \mathrm{kg} / \mathrm{min}$ ) & $214 \pm 185$, day $1 ; 185 \pm 68$, day 3 \\
\hline Ejection fraction (EF, \%) & $62.7 \pm 8.1$, day $1 ; 64.3 \pm 5.8$, day 3 \\
\hline Presence of patent ductus arteriosus & $7 / 7$, day $1 ; 4 / 7$, day 3 \\
\hline Arterial Blood Pressure (ABP, systolic/diastolic) $(\mathrm{mmHg})$ & $55 / 34 \pm 13 / 11$, day $1 ; 57 / 31 \pm 12 / 5$, day 3 \\
\hline Heart Rate (HR, beats per min) & $161 \pm 11$, day $1 ; 156 \pm 8$, day 3 \\
\hline Respiratory Rate (RR, breaths per min) & $59 \pm 11$, day $1 ; 50 \pm 17$, day 3 \\
\hline Arterial oxygen saturation $\left(\mathrm{SaO}_{2}, \%\right)$ & $92.3 \pm 7.3$, day $1 ; 95.5 \pm 2.6$, day 3 \\
\hline
\end{tabular}

Table 1. Patient demographics and clinical metrics.

to inconsistencies in $\mathrm{StO}_{2}$ recordings between oximeters and the fact that $\mathrm{StO}_{2}$ is affected by multiple factors, including CBF, blood oxygen content, and tissue metabolism ${ }^{16,17}$.

Cerebral blood flow can be monitored directly using the flow-sensitive derivative of NIRS, diffuse correlation spectroscopy (DCS $)^{18}$. In turn, the blood flow index (BFi) obtained from DCS can be combined with $\mathrm{StO}_{2}$ to measure an index of the cerebral metabolic rate of oxygen ${ }^{19-25}$. NIRS offers an alternative means of directly assessing metabolism by measuring changes in the oxidation state of cytochrome c oxidase (oxCCO), the terminal enzyme in the mitochondrial electron transport chain. Due to its low tissue concentration relative to hemoglobin, hyperspectral (hs) NIRS is optimal for monitoring oxCCO to avoid crosstalk between chromophores ${ }^{26}$. Combining hsNIRS with DCS offers the ability to evaluate the impact of fluctuations in CBF on cellular oxygen metabolism. Considering that cerebral energy requirements can be maintained during reductions in CBF by a compensatory increase in oxygen extraction ${ }^{27-29}$, detecting concurrent reductions in CBF and oxCCO could be of greater clinical significance-indication of possible hypoxia - than changes in perfusion or oxygenation alone.

With the goal of providing continuous bedside monitoring of CBF and oxCCO, a hybrid hsNIRS/DCS device was built (NNeMo: Neonatal NeuroMonitor) ${ }^{30}$. The aim of this study was to evaluate CBF and oxCCO stability within the first $72 \mathrm{~h}$ of life in preterm infants less than 32 weeks GA and weighing less than $1500 \mathrm{~g}$. It was hypothesized that oxCCO would remain relatively independent of CBF fluctuations due to compensatory changes in oxygen extraction. As a corollary, a stronger temporal correlation between $\mathrm{CBF}$ and $\mathrm{StO}_{2}$ was expected, considering the sizable hemodynamic contributions to $\mathrm{StO}_{2}$.

\section{Results}

Optical data were acquired from nine preterm infants on the first and third days of life. Between 80 and $90 \%$ of parents consented to participate in this study. The first monitoring period began on average at $5.9 \pm 6.2 \mathrm{~h}$ after birth (range: 1 to $18 \mathrm{~h}$ ) and the second at $53.8 \pm 8.2 \mathrm{~h}$ (range: 44.5 to $61 \mathrm{~h}$ ). Data from the first patient were excluded due to excessive ambient light that resulted in substantial signal artifacts. For subsequent acquisitions, the optical probes were covered at the site of contact using a thin blanket to minimize signal from ambient light sources. Data from the remaining eight infants are reported in this study. Average clinical parameters are provided in Table 1 and individual patient values can be found in the supplementary material (Table S1). On average, $233 \pm 68 \mathrm{~min}$ (range: 145 to $340 \mathrm{~min}$ ) of data were analyzed on day one and $254 \pm 36 \mathrm{~min}$ (range: 210 to $290 \mathrm{~min}$ ) on day three. One infant was diagnosed with IVH on the first day: a bilateral bleed with Grade II in the right hemisphere and Grade I on the left. This infant was the only one given inotropic support. All patients survived the neonatal period.

Table 1 also includes cardiac measurements from targeted neonatal echocardiography (TnECHO). Note, TnECHO results are from the first seven patients, as imaging data were not recorded from the last infant due to an archiving error with the echo machine. Two patients had low left ventricular output (i.e. $<150 \mathrm{ml} / \mathrm{kg} / \mathrm{min}$ ) on both days, and one patient had a slightly low ejection fraction $(E F=51 \%)$ on day 1 . No statistically significant change was found between days 1 and 3 for any of the parameters listed in Table 1 .

Average baseline $\mathrm{BF}_{\mathrm{i}}$ was $8.8 \pm 3.6 \times 10^{-9} \mathrm{~cm}^{2} / \mathrm{s}$ on day 1 (range: 4.7 to $16.6 \times 10^{-9} \mathrm{~cm}^{2} / \mathrm{s}$ ) and $16.8 \pm 7.7 \times 10^{-9} \mathrm{~cm}^{2} / \mathrm{s}$ on day 3 (range: 11.1 to $33.0 \times 10^{-9} \mathrm{~cm}^{2} / \mathrm{s}$ ). There was a significant increase in CBF between the two days $(p=0.022)$. Average baseline $\mathrm{StO}_{2}$ was $73.3 \pm 11.9 \%$ on day 1 (range: 54.5 to $85.5 \%$ ) and $80.1 \pm 7.0 \%$ on day 3 (range: 71.9 to $94.5 \%$ ), with no significant difference. Time courses of $\mathrm{StO}_{2}$, relative CBF (rCBF) and change in oxCCO $(\triangle \mathrm{oxCCO})$ on the first day of life for one infant are displayed in Fig. 1 . This figure also 
(a)

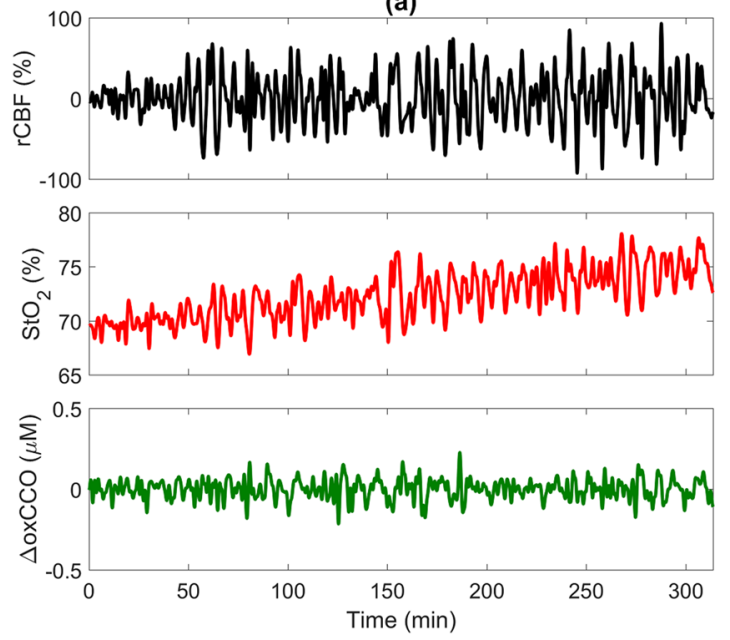

(b)
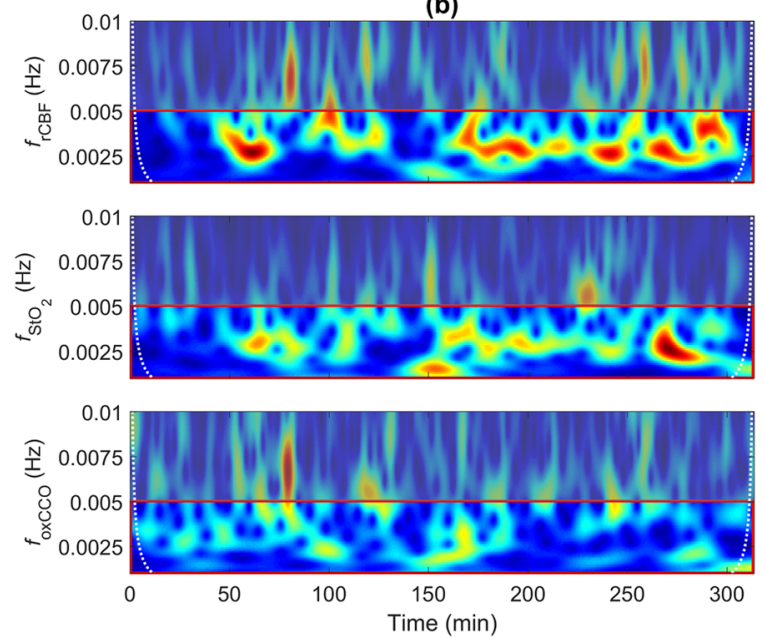

Figure 1. (a) Time courses of relative cerebral blood flow ( $\mathrm{rCBF})$, tissue saturation $\left(\mathrm{StO}_{2}\right)$, and change in the oxidation state of cytochrome $\mathrm{c}$ oxidase $(\triangle \mathrm{oxCCO})$ for one patient on the first day of life. (b) Corresponding frequency-time plots generated by wavelet transform of $\mathrm{rCBF}, \mathrm{StO}_{2}$, and $\Delta$ oxCCO. The red box highlights the frequency band of notable CBF oscillation $(0.001$ to $0.005 \mathrm{~Hz})$. The dotted white lines indicate the cone of influence. Wavelet values outside this region were considered distorted due to edge $\operatorname{artefacts}{ }^{56}$.

(a)

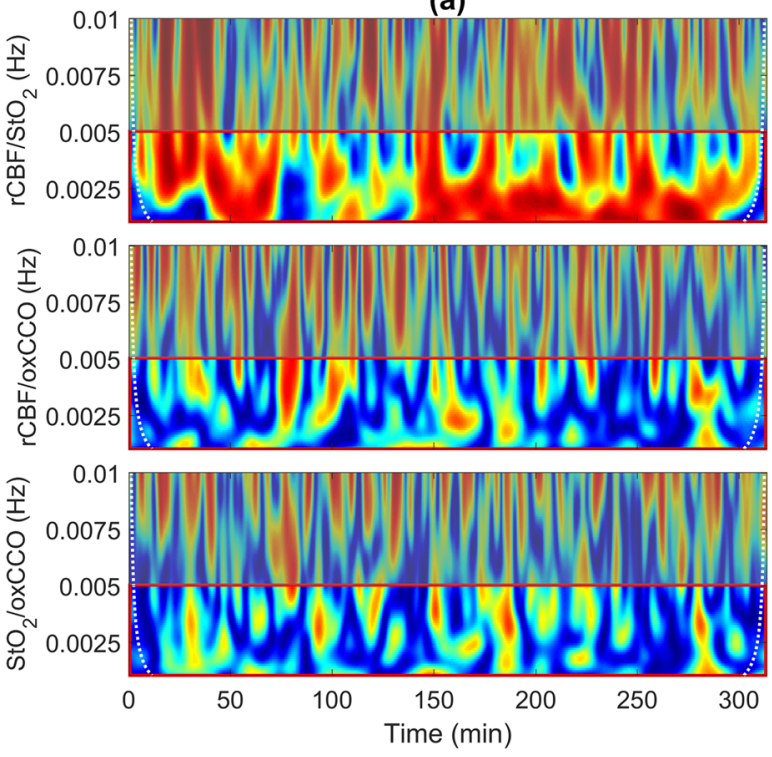

(b)
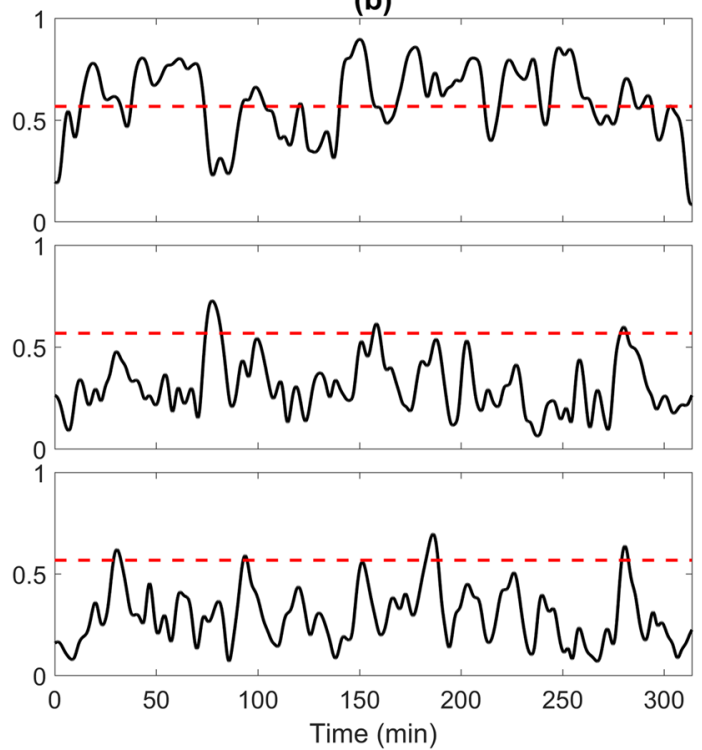

Figure 2. (a) Coherence between relative cerebral blood flow ( $\mathrm{rCBF})$ and tissue saturation $\left(\mathrm{StO}_{2}\right), \mathrm{rCBF}$ and oxidation state of cytochrome c oxidase (oxCCO), and $\mathrm{StO}_{2}$ and oxCCO for one patient on the first day of life. (b) Average coherence value in the frequency range $0.001-0.005 \mathrm{~Hz}$ [indicated by the red box in column (a)]. The red dashed line indicates the statistical threshold.

includes the corresponding wavelet transforms for the three signals. These frequency-time heat maps illustrate the pronounced CBF oscillations in a narrow band of very low frequencies $(0.0025-0.005 \mathrm{~Hz})$ that were fairly consistent across the 5-h period. The standard deviation (SD) of $\mathrm{rCBF}$ across the monitoring period was calculated as a means of characterizing the magnitude of the blood flow oscillations, and in this case, $\mathrm{SD}=32 \%$. These oscillations were also evident in the corresponding $\mathrm{StO}_{2}$ time series but not in $\Delta$ oxCCO. For this patient (see supplementary Table S1 for their clinical characteristics), the hemodynamic oscillations were considerably dampened on the third day ( $\mathrm{SD}=5.7 \%$; data not shown). A general trend of reduced fluctuations in $\mathrm{CBF}$ across participants between the two days was observed ( $\mathrm{SD}=13 \pm 11 \%$ on day 1 versus $8 \pm 7 \%$ on day 3 ); however, this trend was not statistically significant.

Figure 2a displays wavelet coherence of $\mathrm{rCBF} / \mathrm{StO}_{2}, \mathrm{rCBF} / \Delta$ oxCCO and $\mathrm{StO}_{2} / \Delta$ oxCCO for the same patient presented in Fig. 1. Average coherence from 0.001 to $0.005 \mathrm{~Hz}$ is shown in Fig. 2b. For this patient, the coherence between $\mathrm{rCBF}$ and $\mathrm{StO}_{2}$ was above the statistical threshold of 0.57 for $59 \%$ of monitoring period. The corresponding semblance plot (supplementary Fig. S1) indicated that $\mathrm{rCBF}$ and $\mathrm{StO}_{2}$ were predominately in-phase, with a 
(a)

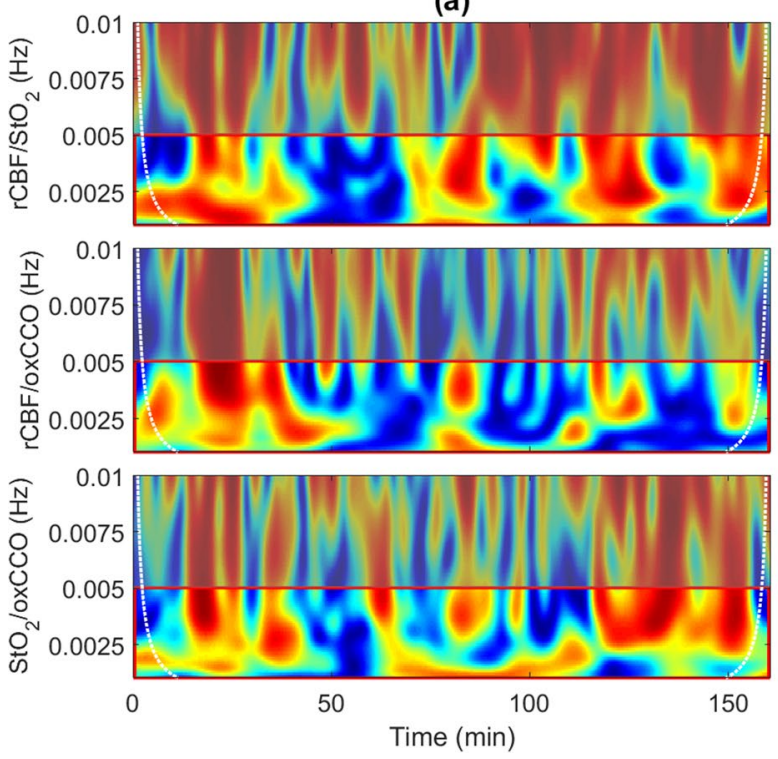

(b)

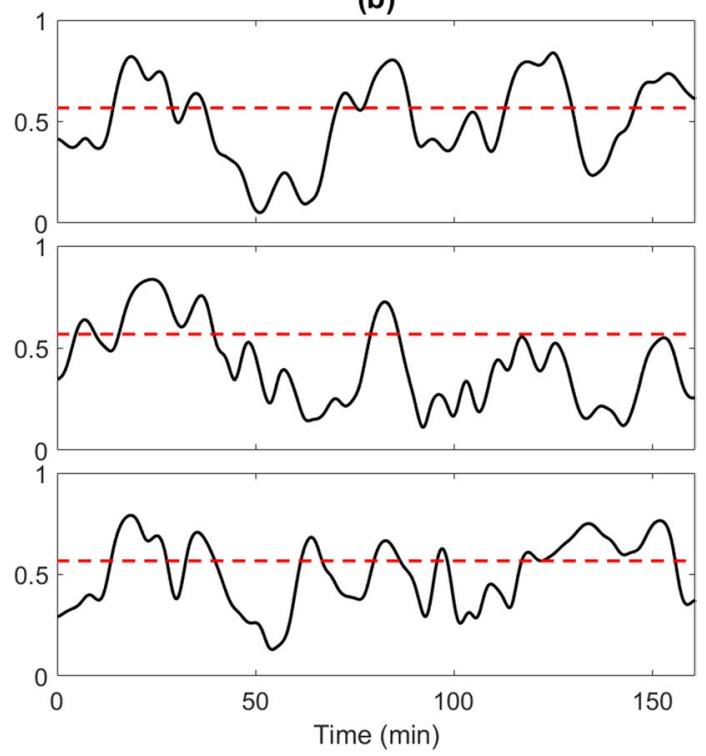

Figure 3. (a) Coherence between $\mathrm{rCBF}$ and $\mathrm{StO}_{2}, \mathrm{rCBF}$ and oxCCO, and $\mathrm{StO}_{2}$ and oxCCO for one patient on the first day of life. (b) Average coherence value in the frequency range $0.001-0.005 \mathrm{~Hz}$. The red dashed line indicates the statistical threshold.

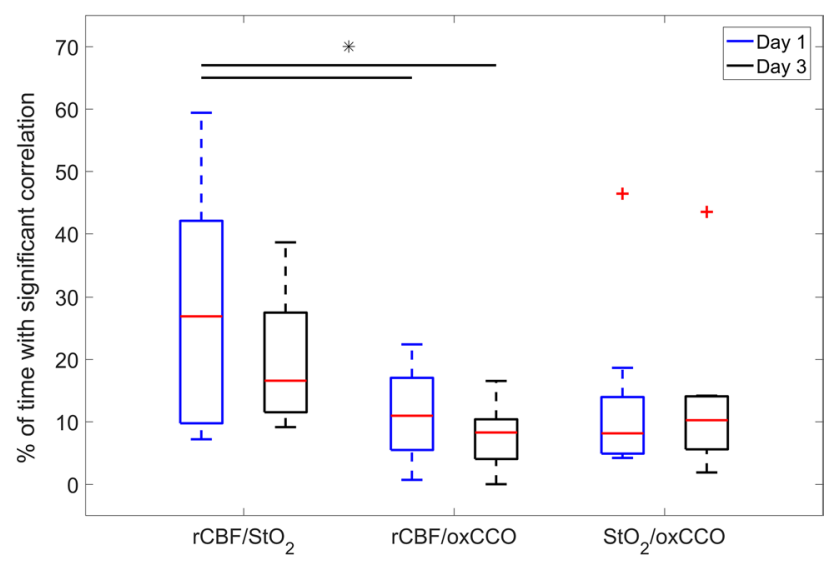

Figure 4. Boxplots of the fraction of time that coherence between two optical parameters reached the statistical threshold of 0.57 . Data are presented separately for the two monitoring periods and for the three combinations of coherence estimates: $\mathrm{rCBF} / \mathrm{StO}_{2}, \mathrm{rCBF} / \Delta$ oxCCO and $\mathrm{StO}_{2} / \Delta$ oxCCO. Statistical outliers are indicated by + , and the bars indicate significant differences in coherence duration between $\mathrm{rCBF} / \mathrm{StO}_{2}$ on day 1 and $\mathrm{rCBF} /$ $\Delta$ oxCCO on days $1(p=0.025)$ and $3(p=0.004)$. Details of each boxplot are as follows: $\mathrm{rCBF} / \mathrm{StO}_{2}($ day 1$)$ : median: 26.9 , 75th percentile: 42.1 , 25 th percentile: $9.8 ; \mathrm{rCBF} / \mathrm{StO}_{2}$ (day 3): median: 16.6 , 75 th percentile: 27.5, 25th percentile: 11.5 ; $\mathrm{rCBF} / \mathrm{oxCCO}$ (day 1): median: 11.0, 75 th percentile: 17.0 , 25th percentile: 5.5 ; $\mathrm{rCBF} / \mathrm{oxCCO}$ (day 3): median: 8.3, 75th percentile: 10.4 , 25th percentile: 4.0 ; $\mathrm{StO}_{2} /$ oxCCO (day 1): median: 8.2; 75 th percentile: $14.0 ; 25$-th percentile: 4.9 ; and $\mathrm{StO}_{2}$ /oxCCO (day 3): median: 10.3 , 75 th percentile: 14.1 , 25th percentile: 5.6 .

mean semblance of $0.56 \pm 0.23$, which was greater than for all other patients (inter-subject mean $=0.27 \pm 0.18$ ). In contrast, the two coherence plots involving $\triangle$ oxCCO only reached significance for $4.5 \%$ and $4.8 \%$ of the monitoring period for $\mathrm{rCBF} / \triangle \mathrm{oxCCO}$ and $\mathrm{StO}_{2} / \triangle$ oxCCO, respectively. Similarly, mean semblance values for this patient were $0.02 \pm 0.34$ for $\mathrm{rCBF} / \Delta \mathrm{oxCCO}$ and $0.05 \pm 0.39$ for $\mathrm{StO}_{2} / \Delta \mathrm{oxCCO}$. For comparison, inter-subject means were $-0.01 \pm 0.1$ and $-0.01 \pm 0.09$ for $\mathrm{rCBF} / \Delta$ oxCCO and $\mathrm{StO}_{2} / \Delta$ oxCCO, respectively.

Figure 3 presents wavelet coherence for another patient who exhibited the greatest coherence with respect to CCO. In this case, data are presented from the monitoring period on day 1 . Coherence was above the statistical threshold for $43 \%$ of the time for $\mathrm{rCBF} / \mathrm{StO}_{2}, 22 \%$ for $\mathrm{rCBF} / \Delta$ oxCCO and $46 \%$ for $\mathrm{StO}_{2} / \Delta$ oxCCO. Similar patterns were also observed on day 3 ; that is, significance coherence was found for $32 \%, 17 \%$ and $44 \%$ of the monitoring period for $\mathrm{rCBF} / \mathrm{StO}_{2}, \mathrm{rCBF} / \Delta$ oxCCO and $\mathrm{StO}_{2} / \Delta$ oxCCO, respectively. 
Figure 4 provides boxplots of the fraction of time that coherence estimates for $\mathrm{rCBF} / \mathrm{StO}_{2}, \mathrm{rCBF} / \Delta \mathrm{oxCCO}$ and $\mathrm{StO}_{2} / \Delta$ oxCCO were greater than the statistical threshold determined from Monte Carlo simulations. Coherence was calculated for the frequency band from 0.001 to $0.005 \mathrm{~Hz}$. A two-way ANOVA revealed that the duration of $\mathrm{rCBF} / \mathrm{StO}_{2}$ coherence on day 1 was significantly greater that the corresponding coherence durations for $\mathrm{rCBF} /$ $\Delta$ oxCCO on both days. The two outliers identified in the $\mathrm{StO}_{2} / \Delta$ oxCCO coherence boxplots are from the patient presented in Fig. 3. If these outliers were excluded, then the $\mathrm{rCBF} / \mathrm{StO}_{2}$ coherence duration on day 1 was also significantly greater that the values for $\mathrm{StO}_{2} / \Delta$ oxCCO on days 1 and 3 .

\section{Discussion}

This proof-of-concept study demonstrated the feasibility of concurrently monitoring cerebral perfusion and metabolism in VLBW preterm infants during the first $72 \mathrm{~h}$ of life. Continuous monitoring was achieved using a hybrid system combining hsNIRS and DCS. While hsNIRS is less common that conventional NIRS system that emit light at a few wavelengths, it has the advantage of providing a direct assessment of oxygen metabolism by measuring oxCCO, and there is an increasing interest in this metabolic marker in neonatal studies ${ }^{31}$. The challenge with combining hsNIRS and DCS is avoiding cross contamination, in particular, blocking the DCS laser that can easily saturate the spectrometer used to measure $\Delta \mathrm{oxCCO}^{28}$. NNeMo avoids crosstalk by incorporating a multiplexing system to collect data from the two subsystems sequentially ${ }^{30}$. The trade-off with this approach is lower temporal resolution compared to hybrid designs that provide simultaneous $\mathrm{CBF}$ and $\mathrm{StO}_{2}$ monitoring using NIRS techniques involving only a few wavelengths ${ }^{21,32,33}$. However, the 7-s duration used to collect DCS and hsNIRS data was sufficient for monitoring very low frequency hemodynamic and metabolic fluctuations (i.e. those less than $0.01 \mathrm{~Hz}$ ).

The primary outcome of the study was to confirm the hypothesis that cerebral metabolism remained relatively independent of cerebral blood flow in the majority $(7 / 8)$ of patients, indicating that adequate oxygen delivery was maintained despite fluctuations in cerebral hemodynamics. Evidence is provided by the low coherence between $\triangle$ oxCCO and both $\mathrm{rCBF}$ and $\mathrm{StO}_{2}$, as shown in Fig. 4. The most dramatic illustration of the independence of oxCCO from CBF is the data set presented in Figs. 1 and 2. This patient exhibited large and sustained oscillations in $\mathrm{rCBF}$ throughout most of monitoring period on the first day. They had the lowest left ventricular output and tachypnea on day 1 (P5 in Table S1), which could contribute to flow instabilities given that impaired cerebral autoregulation is common in preterm infants ${ }^{8}$. Independent of the possible cause, this study demonstrated that the corresponding oxCCO time course exhibited only small variations (less than $0.1 \mu \mathrm{M}$ ) despite large blood flow fluctuations. To put the magnitude of these changes in context, reductions in oxCCO of the order of 1-1.5 $\mu \mathrm{M}$ were reported during desaturation events in term infants with hypoxic-ischemic encephalopathy ${ }^{34}$. The implication of stable oxCCO during variable CBF indicates that this patient was not experiencing cycles of cerebral hypoxia, which has been postulated to contribute to $\mathrm{IVH}^{6}$. In contrast to oxCCO, Fig. 2 illustrates that $\mathrm{StO}_{2}$ was strongly affected by fluctuations in $\mathrm{CBF}$, as indicated by the significant $\mathrm{rCBF} / \mathrm{StO}{ }_{2}$ coherence throughout the five hours. This was confirmed by the $\mathrm{rCBF} / \mathrm{StO}_{2}$ semblance plot (Fig. S1) that showed strong positive correlations between $\mathrm{rCBF}$ and $\mathrm{StO}_{2}$. A positive correlation is expected when there is no change in metabolism since increases in $\mathrm{CBF}$ will cause a greater oxygenation of venous blood and, vice versa, reduced CBF will lower venous oxygenation.

Of the eight patients, only one exhibited hemodynamic/metabolic coherence that was comparable in duration to the coherence between $\mathrm{rCBF}$ and $\mathrm{StO}_{2}$ (Fig. 3; P5 in Table S1). Furthermore, the time series of average coherence for $\mathrm{rCBF} / \mathrm{StO}_{2}, \mathrm{rCBF} / \Delta \mathrm{oxCCO}$ and $\mathrm{StO}_{2} / \Delta$ oxCCO followed similar patterns with good agreement in terms of the specific times of significance coherence. Interestingly, this infant had the lowest baseline BFi, which was $47 \%$ lower than the group average. This finding suggests that cerebral metabolism is less tolerant to hemodynamic instabilities in patients with low baseline CBF, presumably because at low flow the brain is close to the lower threshold of sufficient oxygen delivery. More studies are required to confirm this postulate considering this patient did not develop IVH. In all likelihood, any link between flow/metabolic coherence and the development of brain injury shortly after birth will depend on the duration of coherence and the magnitude of CBF changes. It would also be useful in future studies to acquire continuous recordings of $\mathrm{ABP}$ and $\mathrm{SaO}_{2}$ to investigate which clinical features are likely driving the $\mathrm{rCBF} / \triangle$ oxCCO coherence. Nevertheless, these preliminary results highlight the value of directly monitoring CBF with DCS, as opposed to relying on $\mathrm{StO}_{2}$ as a surrogate marker. It should be noted that although DCS does not measure CBF in classic units of perfusion, BFi has been shown to closely track $\mathrm{CBF}$ in both neonatal animal models and human infants ${ }^{24,35-38}$.

Average $\mathrm{StO}_{2}$ values $(73.3 \pm 11.9 \%$ on day 1 and $80.1 \pm 7.0 \%$ on day 3$)$ were comparable to values reported by Roche-Labarbe et al. (between 75 to $83 \%$ ) for preterm infants less than a GA $<30$ weeks and Noori et al. (mean of $79 \%$ ) for infants less than 27 weeks of age ${ }^{20,39}$. However, a larger study reported average $\mathrm{StO}_{2}$ closer to $65 \%$ during the first $72 \mathrm{~h}$ of life for preterm infants ${ }^{40}$. Discrepancies are likely related to differences in NIRS devices (hsNIRS in the current study, frequency-domain NIRS in the Roche-Labarbe study, and continuous-wave systems in the other two studies), source-detector distances, and patient populations. Similar to previous studies ${ }^{39,40}$, we found no significant difference in $\mathrm{StO}_{2}$ between the two monitoring periods that were 6 and $54 \mathrm{~h}$ after birth, respectively. In contrast, a significant increase in CBF of $45 \%$ was found between the two monitoring periods. To the best of our knowledge, this is the first study to monitor cerebral perfusion directly during the first $72 \mathrm{~h}$ of life in preterm infants. The mean baseline BFi on day $3\left(16.8 \pm 7.7 \times 10^{-9} \mathrm{~cm}^{2} / \mathrm{s}\right)$ was in good agreement with a previous DCS study involving preterm infants ${ }^{41}$, but lower than the value reported for healthy term infants $\left(27.1 \pm 15.6 \times 10^{-9} \mathrm{~cm}^{2} / \mathrm{s}\right)^{42}$. This difference could be due to brain maturation, although BFi estimates will also be affected by the chosen $\mu_{a}$ and $\mu_{s}^{\prime}$ values.

As a feasibility study, the sample size was too small to assess the relationship between flow/metabolism coupling and the occurrence of IVH. In fact, only one patient was diagnosed with Grade 1 IVH, which was detected 
(a)

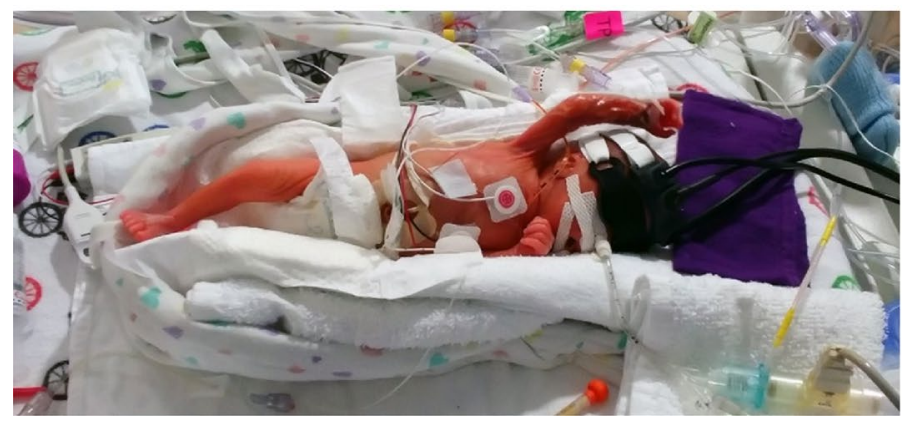

(b)

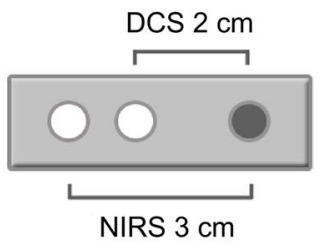

Figure 5. (a) Premature infant with optical probes secured to the forehead and a phototherapy eye shield, (b) schematic of probe holder showing the position of the NIRS (3-cm SDD) and DCS (2-cm SDD) sources and the common detection location (shaded circle). Probe holder was $5 \times 2 \times 1 \mathrm{~cm}$.

by cUS at the beginning of the monitoring period on day 1 (P3 in Table S1). This patient exhibited similar stability metrics compared to the rest of the patients with a relative duration of significant $\mathrm{rCBF} / \mathrm{StO}_{2}$ coherence of $41 \%$ and a corresponding rCBF/oxCCO coherence of $12 \%$. Despite the limited sample size, the study demonstrated that monitoring $\mathrm{CBF}, \mathrm{StO}_{2}$ and oxCCO could begin shortly after birth and continue for extended periods. A monitoring period of $6 \mathrm{~h}$ was selected for this initial study; however, the amount of usable data was reduced to $233 \pm 68 \mathrm{~min}$ on the first day and $254 \pm 36 \mathrm{~min}$ on day three due to signal artefacts related primarily to patient handling. No evidence of skin irritation caused by the probe holder was found in any of the sessions, indicating that continuously monitoring throughout the first $72 \mathrm{~h}$ is achievable.

An alternative to measuring oxCCO is to calculate the cerebral metabolic rate of oxygen from $\mathrm{CBF} \mathrm{SaO}_{2}$ and $\mathrm{StO}_{2}$ measurements, which has the advantage that $\mathrm{StO}_{2}$ can be measured by more common NIRS technologies than hsNIRS ${ }^{19-25}$. Despite measuring $\mathrm{CBF}$ and $\mathrm{StO}_{2}$ in the current study, a comparison between these two metabolic markers was not conducted as the required $\mathrm{SaO}_{2}$ data could not be retrieved from the clinical monitors. This study limitation highlights a potential advantage to measuring oxCCO since it is a direct marker of metabolism that does not require other input parameters. Clinical interest in oxCCO monitoring will likely increase given the feasibility of using low-cost spectrometers ${ }^{43,44}$.

In summary, this is the first study to demonstrate how continuous cerebral perfusion and metabolic monitoring in VLBW preterm infants can be performed by combining hsNIRS and DCS. The application of wavelet coherence analysis demonstrated that the temporal correlation between $\mathrm{StO}_{2}$ and $\mathrm{rCBF}$ on the first day of life was significantly greater than the corresponding correlation between $\triangle$ oxCCO and either $\mathrm{rCBF}_{\mathrm{f}} \mathrm{StO} \mathrm{S}_{2}$. This finding indicates that cerebral oxygen metabolism is generally independent of hemodynamic fluctuations. However, significant coherence between $\triangle$ oxCCO and rCBF was found in the patient with the lowest baseline blood flow index, suggesting that the combination of low cerebral blood flow and hemodynamic instability can affect metabolism. Further studies are required to determine if $\mathrm{CBF} /$ oxCCO monitoring could help identify preterm infants at greater risk of IVH.

\section{Methods}

Patient population. This study was approved by the Western University Health Sciences Research Ethics Board, which adheres to the guidelines of the Canadian Tri-Council Policy Statement: Ethical Conduct for Research Involving Humans in accordance with the Declaration of Helsinki. Patient recruitment was conducted in the neonatal intensive care unit at the Children's Hospital, London Health Sciences Center. Informed -parental consent was obtained for all patients recruited for this study. Participants were infants born less than 32 weeks GA and weighing less than $1500 \mathrm{~g}$. Neuromonitoring consisted of two periods, each up to $6 \mathrm{~h}$ in duration, that were within the first $72 \mathrm{~h}$ of life. The first period started as soon as clinically feasible following birth and resuscitation, and the second period started at $48 \mathrm{~h}$ post-natal age. In each session, cUS was performed to diagnose cerebral hemorrhaging, which was graded according to the Papile scale ${ }^{45}$ and targeted neonatal echocardiography (TnECHO) used to assess cardiac output (i.e. left ventricular output and ejection fraction) and to screen for patent ductus arteriosus ${ }^{46}$. Heart rate $(\mathrm{HR})$, respiratory rate $(\mathrm{RR})$ and arterial oxygen saturation $\left(\mathrm{SaO}_{2}\right)$ were recorded from the clinical monitor at the beginning and end of each period. Note, the monitors used in the unit did not provide the option to save the continuous recordings of these parameters. Arterial blood pressure (ABP) was measured non-invasively at the beginning of each period.

Study design. Once transferred to the NICU from the birthing suite and following initial vitals by nursing staff, the optical probes were secured to the scalp above the frontoparietal cortex using a 3-D printed probe holder and adjustable strap (Fig. 5A). The optical fibers were bent $90^{\circ}$ at the point of contact on the head for ease of use, and the optical prober holder was designed to be light, flexible and non-abrasive (Fig. 5B; dimension of $5 \times 2 \times 1 \mathrm{~cm}$; Flexible Resin, Form 2, Formlabs, Somerville, MA, USA). The design of the probe holder enabled it to be used with infants requiring ventilation with continuous positive airway pressure (CPAP) as it could be positioned under the CPAP cap and tube. The infant's eyes were shielded using phototherapy eye goggles as a 
precaution. Two optical fiber bundles (hsNIRS and DCS sources) directed light to the head, while a third (common detection) collected diffusely reflected light. The source-detector distance was $3 \mathrm{~cm}$ for hsNIRS and $2 \mathrm{~cm}$ for DCS. A smaller distance was chosen for DCS as it inherently has greater sensitivity to the brain ${ }^{47}$. Power levels for the two light sources and spot size incident on the scalp were adjusted to meet ANSI standards for skin exposure. Continuous DCS and hsNIRS data sets were saved in one-hour intervals throughout each monitoring period. One member of the research team remained at the bedside during data acquisition to log any events or clinical procedures that could affect the quality of the optical signals. Following each monitoring period, the probe holder was removed, and the skin was assessed for redness or irritation. This workflow was repeated for the monitoring session on the third day.

Instrumentation. The NNeMo system combines hsNIRS and DCS by incorporates a multiplexing shuttering system to cycle between acquisitions from the two subsystems ${ }^{29,30}$. For this study, hsNIRS and DCS data were each acquired at a sampling frequency of $4 \mathrm{~Hz}$ in consecutive 3-s intervals, resulting in full data sets every $7 \mathrm{~s}$ (with a 0.5 -s dead-time between subsystems). The hsNIRS system employed a 20-W halogen bulb filtered outside of 600 to $1000 \mathrm{~nm}$ (Hl-2000-HP, Ocean Insight, Delay Beach, FL, USA) and a spectrometer (iDus 420, Andor, Oxford Instruments, Abingdon, UK; 548-1085 nm bandwidth; $1.65 \mathrm{~nm}$ resolution; P\&P Optica, Waterloo, $\mathrm{ON}$, Canada). An optical fiber bundle $(2.4 \mathrm{~mm}$ outer diameter, core $=30 \mu \mathrm{M}$, numerical aperture (NA) $=0.55)$, Loptek, Berlin, Germany) directed light towards the scalp, while a second set of fibers (three linearly aligned fibers; diameter $=2 \mathrm{~mm}$, core $=30 \mu \mathrm{m}, \mathrm{NA}=0.55$, Loptek) collected diffusely reflected light. The DCS system consisted of a long-coherence laser (DL785-100-S, CrystaLaser, Reno, NV, USA) and a four-channel single-photon counting module (SPCM-AQR-15-FC, Excelitas Technologies, Montreal, QC, Canada). The laser was coupled to four fibers (core $=200 \mu \mathrm{m}, \mathrm{NA}=0.22$, Loptek), and four single-mode fibers were used for detection (core $=8 \mu \mathrm{m}$, $\mathrm{NA}=0.12$, Loptek). The output for the SPCM was fed into a PCIe-6612 data acquisition board that generated intensity autocorrelation functions using in-house developed software: LabVIEW 2017 SP1 (National Instruments, https://www.ni.com/en-ca/support/downloads/software products/download.labview.html\#306,351) and MATLAB 2016b (MathWorks, https://www.mathworks.com/help/releases/R2016b/index.html ${ }^{48}$.

Data analysis. Quantifying $\mathrm{StO}_{2}$ and changes in oxCCO by hsNIRS. Each monitoring period began by collecting a dark spectrum $\left(\operatorname{dark}_{\lambda}\right)$ acquired with the emission source turned off. A reference spectrum (reference $\left.{ }_{\lambda}\right)$ was collected to characterize the spectral properties intrinsic to the instrument. Each reflectance spectrum, $R(\lambda)$, was determined by: ${ }^{49}$

$$
R(\lambda)=\frac{\operatorname{spectrum}_{\lambda}-\operatorname{dark}_{\lambda}}{\text { reference }_{\lambda}-\operatorname{dark}_{\lambda}}
$$

where spectrum $_{\lambda}$ refers to intensity measurements as a function of wavelength $\lambda$.

A derivative spectroscopy approach was applied to $R(\lambda)$ to quantify the tissue water fraction (WF) and baseline concentrations of oxy- and deoxy-hemoglobin $\left(\mathrm{HbO}_{2}^{b} \text { and } \mathrm{Hb}^{b} \text {, respectively }\right)^{24,50}$. The approach involves fitting the first and second derivatives of $\mathrm{R}(\lambda)$ with the solution to the diffusion approximation for a semi-infinite homogeneous medium. Light absorption and scattering parameters were input into the model solution as follows:

$$
\begin{gathered}
\mu_{a}(\lambda)=W F \cdot \varepsilon_{H_{2} O}(\lambda)+H b^{b} \cdot \varepsilon_{H b}(\lambda)+H b O_{2}^{b} \cdot \varepsilon_{H b O_{2}}(\lambda) \\
\mu_{s}^{\prime}(\lambda)=A \cdot\left(\frac{\lambda}{800 \mathrm{~nm}}\right)^{-\alpha}
\end{gathered}
$$

where $\mu_{a}(\lambda)$ is the absorption coefficient, $\varepsilon$ refers to the molar extinction coefficient of each chromophore, $\mu_{s}^{\prime}(\lambda)$ is the reduced scattering coefficient, $\alpha$ is the scattering power, and A is the value of $\mu_{s}^{\prime}(\lambda)$ at $\lambda=800 \mathrm{~nm}$.

Following baseline analysis, a modified Beer-Lambert Law approach based on the UCLn algorithm was utilized to determine time-varying changes in the concentrations of $\mathrm{HbO}_{2}, \mathrm{Hb}$ and oxCCO${ }^{26}$ :

$$
\left[\begin{array}{c}
\Delta H b O_{2}(t) \\
\Delta H b(t) \\
\Delta o x C C O(t)
\end{array}\right]=\frac{1}{D P}\left[\begin{array}{ccc}
\varepsilon_{H b O_{2}}\left(\lambda_{1}\right) & \varepsilon_{H b}\left(\lambda_{1}\right) & \varepsilon_{o x C C O}\left(\lambda_{1}\right) \\
\vdots & \vdots & \vdots \\
\varepsilon_{H_{b O_{2}}}\left(\lambda_{n}\right) & \varepsilon_{H b}\left(\lambda_{n}\right) & \varepsilon_{o x C C O}\left(\lambda_{1}\right)
\end{array}\right] \cdot\left[\begin{array}{c}
\Delta A\left(\lambda_{1}, t\right) \\
\vdots \\
\Delta A\left(\lambda_{n}, t\right)
\end{array}\right]
$$

where $\Delta$ denotes a change relative to baseline concentration, DP is the differential pathlength (set to 4.9 based on previous literature ${ }^{51}$ ), and $\mathrm{A}$ is the measured change in attenuation. $\mathrm{StO}_{2}$ as a function of time was determined by:

$$
S t \mathrm{O}_{2}=\frac{\mathrm{HbO}_{2}^{b}+\Delta \mathrm{HbO}_{2}(t)}{\mathrm{HbO}_{2}^{b}+\Delta \mathrm{HbO}_{2}(t)+\mathrm{Hb}^{b}+\Delta \mathrm{Hb}(t)}
$$

Monitoring CBF by DCS. To determine $\mathrm{BF}_{\mathrm{i}}$, each normalized intensity autocorrelation curve was converted to electric field autocorrelation data using the Siegert relation ${ }^{18}$ :

$$
g_{2}(\rho, \tau)=1+\beta \frac{\left|G_{1}(\rho, \tau)\right|^{2}}{I(\rho, \tau)^{2}}
$$


where $\mathrm{g}_{2}(\rho, \tau)$ represents the measured normalized intensity autocorrelation as a function of source-detector distance $(\rho)$ and correlation time $(\tau), G_{1}(\rho, \tau)$ is the electric field autocorrelation function, $<I(\rho, \tau)>$ is the average intensity, and $\beta$ is the coherence factor. $G_{1}$ was fit with the solution to the diffusion approximation for a semiinfinite homogenous medium based on the assumption of pseudo-Brownian motion of light scatterers ${ }^{52,53}$. The fitting was performed by incorporating changes in $\mu_{a}(\lambda)$ determined by hsNIRS and assuming $\mu_{s}^{\prime}(\lambda)=8 \mathrm{~cm}^{-1}$. The average $\mathrm{BF}_{\mathrm{i}}$ for the first $15 \mathrm{~min}$ of data acquisition was used to define the baseline $\overline{B F}_{i}$. All $\mathrm{BF}_{\mathrm{i}}$ values were normalized to $\overline{B F}_{i}$ to generate a time series of fractional changes in CBF: $r C B F=\left(B F_{i}-\overline{B F}_{i}\right) / \overline{B F}_{i}$.

Data processing and statistical analysis. The first step was to remove data sections that exhibited substantial signal artifacts. The criteria were based on large signal variations in either a hemoglobin time course or the corresponding blood flow index that exceeded four times the temporal standard deviation and persisted for at least $15 \mathrm{~min}$. A comparison to the clinical log was conducted to establish the most likely cause of each artifact (see supplementary Fig. S2 as an example). The most common cause, which occurred at least once in every monitoring period, was patient handling related to checking vital signs, patient repositioning, and responding to desaturation events (i.e., a decrease in $\mathrm{SaO}_{2}$ below 85\%). In addition, six periods were disrupted by clinical procedures including cUS and $x$-ray imaging, phototherapy and surfactant treatment. Data corresponding to any identified artifact were subsequently removed from all time courses. The second step was to filter the $\mathrm{StO}_{2}$, $\mathrm{rCBF}$, and $\triangle \mathrm{oxCCO}$ time courses using an inverse wavelet transform with a Morlet wavelet (the MATLAB function $c w t)^{54-56}$ to remove frequencies greater than $0.01 \mathrm{~Hz}$ and less than $0.001 \mathrm{~Hz}$. The final step was to correct each time course for motion artifacts using an algorithm that utilizes a moving standard deviation and spline interpolation ${ }^{57}$.

Absolute baseline measurements of $\mathrm{BF}_{\mathrm{i}}$ and $\mathrm{StO}_{2}$ were determined for each monitoring period by averaging across the first 15 min of data acquisition. A paired t-test was used to compare baseline $\mathrm{BF}_{\mathrm{i}}$ and $\mathrm{StO}_{2}$ values from the two periods, as well as all other physiological parameter measured on days 1 and 3. All statistical analysis was conducted using a statistical toolbox (MATLAB R2020b) and significance was defined as $p<0.05$. All data are presented as mean \pm standard deviation.

Wavelet coherence, which has been used in a number of neonatal brain monitoring studies ${ }^{23,58-60}$, was used to assess the temporal correlation between the three dynamic signals across the frequency range 0.001 to $0.005 \mathrm{~Hz}$ : $\mathrm{rCBF}$ and $\mathrm{StO}_{2}, \mathrm{rCBF}$ and $\triangle \mathrm{oxCCO}$, and $\mathrm{StO}_{2}$ and $\triangle \mathrm{oxCCO}$. Coherence ranges from 0 to 1 and reflects the crosscorrelation between two time series as a function of frequency, with a value of 0 indicating no correlation and 1 indicating complete agreement. Statistical significance coherence values were determined from Monte Carlo simulations of 1000 data pairs of simulated red noise. Coherence was computed for each pair, and the statistical threshold for the experimental data was based on values greater than $95 \%$ of the simulated values ${ }^{56}$. The final step was to calculate the duration of significance coherence for $\mathrm{rCBF} / \mathrm{StO}_{2}, \mathrm{rCBF} / \Delta$ oxCCO and $\mathrm{StO}_{2} / \Delta$ oxCCO for each patient and monitoring session. A two-way analysis of variance (ANOVA) was used to investigate differences in coherence durations for $\mathrm{rCBF} / \mathrm{StO}{ }_{2}, \mathrm{rCBF} / \Delta$ oxCCO and $\mathrm{StO}_{2} / \Delta$ oxCCO from the two sessions. Boxplots were used to display the results with statistical outliers determined as points greater than $\mathrm{q} 3+\mathrm{w} \times(\mathrm{q} 3-\mathrm{q} 1)$ or less than $\mathrm{q} 1-\mathrm{w} \times(\mathrm{q} 3-\mathrm{q} 1)$ where $\mathrm{q}$ is the quartile number and $\mathrm{w}$ is the whisker length.

To assess the phasic relationship between the signals, wavelet semblance was calculated ${ }^{55}$. Semblance is the instantaneous phase difference and ranges from - 1 for signals completely out of phase to 1 for signals in-phase.

Received: 12 June 2021; Accepted: 2 December 2021

Published online: 07 January 2022

\section{References}

1. Nosarti, C. et al. Adolescents who were born very preterm have decreased brain volumes. Brain 125, 1616-1623. https://doi.org/ 10.1093/brain/awf157 (2002).

2. Stewart, A. L. et al. Ultrasound appearance of the brain in very preterm infants and neurodevelopmental outcome at 18 months of age. Arch. Dis. Child. 58, 598-604. https://doi.org/10.1136/adc.58.8.598 (1983).

3. Volpe, J. J. Brain injury in premature infants: a complex amalgam of destructive and developmental disturbances. Lancet Neurol. 8, 110-124. https://doi.org/10.1016/S1474-4422(08)70294-1 (2009).

4. Bassan, H. Intracranial hemorrhage in the preterm infant: understanding it, preventing it. Clin. Perinatol. 36, 737-762. https:// doi.org/10.1016/j.clp.2009.07.014 (2009).

5. McCrea, H. J. \& Ment, L. R. The diagnosis, management and postnatal prevention of IVH in the preterm neonate. Clin. Perinatol. https://doi.org/10.1016/j.clp.2008.07.014(2010).

6. Vesoulis, Z. A. \& Mathur, A. M. Cerebral autoregulation, brain injury, and the transitioning premature infant. Front. Pediatr. 5, 64. https://doi.org/10.3389/fped.2017.00064 (2017).

7. Volpe, J. J., Kinney, H. C., Jensen, F. E. \& Rosenberg, P. A. The developing oligodendrocyte: key cellular target in brain injury in the premature infant. Int. J. Dev. Neurosci. 29, 423-440. https://doi.org/10.1016/j.ijdevneu.2011.02.012 (2011).

8. Soul, J. S. et al. Fluctuating pressure-passivity is common in the cerebral circulation of sick premature infants. Pediatr. Res. 61, 467-473. https://doi.org/10.1203/pdr.0b013e31803237f6 (2007).

9. Altman, D. I. et al. Cerebral blood flow requirement for brain viability in newborn infants is lower than in adults. Ann. Neurol. 24, 218-226. https://doi.org/10.1002/ana.410240208 (1988).

10. Greisen, G., Andresen, B., Plomgaard, A. M. \& Hyttel-Sørensen, S. Cerebral oximetry in preterm infants: An agenda for research with a clear clinical goal. Neurophotonics 3, 031407. https://doi.org/10.1117/1.nph.3.3.031407 (2016).

11. Wolf, M. \& Greisen, G. Advances in near-infrared spectroscopy to study the brain of the preterm and term neonate. Clin. Perinatol. 36, 807-834. https://doi.org/10.1016/j.clp.2009.07.007 (2009).

12. Dix, L. M. L., van Bel, F. \& Lemmers, P. M. A. Monitoring cerebral oxygenation in neonates: An update. Front. Pediatr. https://doi. org/10.3389/fped.2017.00046 (2017). 
13. Pellicer, A. et al. The SafeBoosC phase II randomised clinical trial: A treatment guideline for targeted near-infrared-derived cerebral tissue oxygenation versus standard treatment in extremely preterm infants. Neonatology 104, 171-178. https://doi.org/10.1159/ 000351346 (2013).

14. Plomgaard, A. M. et al. No neurodevelopmental benefit of cerebral oximetry in the first randomised trial (SafeBoosC II) in preterm infants during the first days of life. Acta Paediatr. Int. J. Paediatr. 108, 275-281. https://doi.org/10.1111/apa.14463 (2019).

15. Hyttel-Sorensen, S., Greisen, G., Als-Nielsen, B. \& Gluud, C. Cerebral near-infrared spectroscopy monitoring for prevention of brain injury in very preterm infants. Cochrane Database Syst. Rev. https://doi.org/10.1002/14651858.CD011506.pub2 (2017).

16. Kleiser, S. et al. Comparison of tissue oximeters on a liquid phantom with adjustable optical properties: an extension. Biomed. Opt. Exp. 9, 86-101. https://doi.org/10.1364/boe.9.000086 (2018).

17. Boas, D. A. \& Franceschini, M. A. Haemoglobin oxygen saturation as a biomarker: the problem and a solution. Philos. Trans. A. Math. Phys. Eng. Sci. 369, 4407-4424. https://doi.org/10.1098/rsta.2011.0250 (2011).

18. Durduran, T. \& Yodh, A. G. Diffuse correlation spectroscopy for non-invasive, micro-vascular cerebral blood flow measurement. Neuroimage 85(1), 51-63. https://doi.org/10.1016/j.neuroimage.2013.06.017 (2014).

19. Roche-labarbe, N. et al. Noninvasive optical measures of CBV, StO2, CBF index, and rCMRO2 in human premature neonates' brains in the first six weeks of life. Hum. Brain Mapp. 31, 341-352. https://doi.org/10.1002/hbm.20868. Noninvasive (2010).

20. Roche-Labarbe, N. et al. Near-infrared spectroscopy assessment of cerebral oxygen metabolism in the developing premature brain. J. Cereb. Blood Flow Metab. 32, 481-488. https://doi.org/10.1038/jcbfm.2011.145 (2012).

21. Dehaes, M. et al. Cerebral oxygen metabolism in neonatal hypoxic ischemic encephalopathy during and after therapeutic hypothermia. J. Cereb. Blood Flow Metab. 34, 87-94. https://doi.org/10.1038/jcbfm.2013.165 (2014).

22. Buckley, E. M., Parthasarathy, A. B., Grant, P. E., Yodh, A. G. \& Franceschini, M. A. Diffuse correlation spectroscopy for measurement of cerebral blood flow: future prospects. Neurophotonics 1, 011009. https://doi.org/10.1117/1.NPh.1.1.011009 (2014).

23. Nourhashemi, M., Mahmoudzadeh, M., Goudjil, S., Kongolo, G. \& Wallois, F. Neurovascular coupling in the developing neonatal brain at rest. Hum. Brain Mapp. 41, 503-519. https://doi.org/10.1002/hbm.24818 (2020).

24. Diop, M., Kishimoto, J., Toronov, V., Lee, D. S. C. \& St Lawrence, K. Development of a combined broadband near-infrared and diffusion correlation system for monitoring cerebral blood flow and oxidative metabolism in preterm infants. Biomed. Opt. Exp. 6, 3907-3918. https://doi.org/10.1364/BOE.6.003907 (2015).

25. Lin, P. Y., Hagan, K., Fenoglio, A., Grant, P. E. \& Franceschini, M. A. Reduced cerebral blood flow and oxygen metabolism in extremely preterm neonates with low-grade germinal matrix- intraventricular hemorrhage. Sci. Rep. 1, 1. https://doi.org/10.1038/ srep25903 (2016).

26. Bale, G., Elwell, C. E. \& Tachtsidis, I. From Jöbsis to the present day: a review of clinical near-infrared spectroscopy measurements of cerebral cytochrome-c-oxidase. J. Biomed. Opt. 21, 091307. https://doi.org/10.1117/1.JBO.21.9.091307 (2016).

27. Cooper, J. A. et al. Continuous monitoring of absolute cerebral blood flow by near-infrared spectroscopy during global and focal temporary vessel occlusion. J. Appl. Physiol. 110, 1691-1698. https://doi.org/10.1152/japplphysiol.01458.2010 (2011).

28. Rajaram, A. et al. Simultaneous monitoring of cerebral perfusion and cytochrome c oxidase by combining broadband near-infrared spectroscopy and diffuse correlation spectroscopy. Biomed. Opt. Exp. 9, 2588-2603. https://doi.org/10.1364/BOE.9.002588 (2018).

29. Rajaram, A. et al. Optical monitoring of cerebral perfusion and metabolism in adults during cardiac surgery with cardiopulmonary bypass. Biomed. Opt. Exp. 11, 5967-5981. https://doi.org/10.1364/boe.404101 (2020).

30. Rajaram, A. et al. Perfusion and metabolic neuromonitoring during ventricular taps in infants with post-hemorrhagic ventricular dilatation. Brain Sci. 10, 1-13. https://doi.org/10.3390/brainsci10070452 (2020).

31. Harvey-Jones, K., Lange, F., Tachtsidis, I., Robertson, N. J. \& Mitra, S. Role of optical neuromonitoring in neonatal encephalopathy-current state and recent advances. Front. Pediatr. 9, 1-18. https://doi.org/10.3389/fped.2021.653676 (2021).

32. Milej, D. et al. Characterizing dynamic cerebral vascular reactivity using a hybrid system combining time-resolved near-infrared and diffuse correlation spectroscopy. Biomed. Opt. Express 11, 4571-4585. https://doi.org/10.1364/boe.392113 (2020).

33. Giovannella, M. BabyLux device: A diffuse optical system integrating diffuse correlation spectroscopy and time-resolved nearinfrared spectroscopy for the neuromonitoring of the premature newborn brain. Neurophotonics. https://doi.org/10.1117/1.nph.6. 2.025007 (2019).

34. Bale, G. et al. Oxygen dependency of mitochondrial metabolism indicates outcome of newborn brain injury. J. Cereb. Blood Flow Metab. https://doi.org/10.1177/0271678X18777928 (2018).

35. Jain, V. et al. Cerebral oxygen metabolism in neonates with congenital heart disease quantified by MRI and optics. J. Cereb. Blood Flow Metab. 34, 380-388. https://doi.org/10.1038/jcbfm.2013.214 (2014).

36. Buckley, E. M. et al. Validation of diffuse correlation spectroscopic measurement of cerebral blood flow using phase-encoded velocity mapping magnetic resonance imaging. J. Biomed. Opt. 17, 037007. https://doi.org/10.1117/1.JBO.17.3.037007 (2012).

37. Giovannella, M. et al. Validation of diffuse correlation spectroscopy against 15O-water PET for regional cerebral blood flow measurement in neonatal piglets. J. Cereb. Blood Flow Metab. 40, 2055-2065. https://doi.org/10.1177/0271678X19883751 (2020).

38. Diop, M., Verdecchia, K., Lee, T.-Y. \& St Lawrence, K. Calibration of diffuse correlation spectroscopy with a time-resolved nearinfrared technique to yield absolute cerebral blood flow measurements. Biomed. Opt. Exp. 2, 2068-2081. https://doi.org/10.1364/ BOE.2.002068 (2011).

39. Noori, S., McCoy, M., Anderson, M. P., Ramji, F. \& Seri, I. Changes in cardiac function and cerebral blood flow in relation to peri/ intraventricular hemorrhage in extremely preterm infants. J. Pediatr. 164, 264-270. https://doi.org/10.1016/j.jpeds.2013.09.045 (2014).

40. Alderliesten, T. et al. Reference values of regional cerebral oxygen saturation during the first 3 days of life in preterm neonates. Pediatr. Res. 79, 55-64. https://doi.org/10.1038/pr.2015.186 (2016).

41. Buckley, E. M. et al. Cerebral hemodynamics in preterm infants during positional intervention measured with diffuse correlation spectroscopy and transcranial Doppler ultrasound. Opt. Exp. 17, 12571-12581 (2009).

42. Andresen, B. et al. Cerebral oxygenation and blood flow in normal term infants at rest measured by a hybrid near-infrared device (BabyLux). Pediatr. Res. https://doi.org/10.1038/s41390-019-0474-9 (2019).

43. Diop, M., Wright, E., Toronov, V., Lee, T.-Y. \& St Lawrence, K. Improved light collection and wavelet de-noising enable quantification of cerebral blood flow and oxygen metabolism by a low-cost, off-the-shelf spectrometer. J. Biomed. Opt. 19, 057007. https:// doi.org/10.1117/1.JBO.19.5.057007 (2014).

44. Kaynezhad, P. et al. Quantification of the severity of hypoxic-ischemic brain injury in a neonatal preclinical model using measurements of cytochrome-c-oxidase from a miniature broadband-near-infrared spectroscopy system. Neurophotonics 6, 1. https://doi. org/10.1117/1.nph.6.4.045009 (2019).

45. Cherian, S., Whitelaw, A., Thoresen, M. \& Love, S. The pathogenesis of neonatal post-hemorrhagic hydrocephalus. Brain Pathol. 14, 305-311. https://doi.org/10.1111/j.1750-3639.2004.tb00069.x (2004).

46. Mertens, L. et al. Targeted neonatal echocardiography in the neonatal intensive care unit: Practice guidelines and recommendations for training. Eur. J. Echocardiogr. 24, 1057-1078. https://doi.org/10.1093/ejechocard/jer181 (2011).

47. Selb, J. et al. Sensitivity of near-infrared spectroscopy and diffuse correlation spectroscopy to brain hemodynamics: simulations and experimental findings during hypercapnia. Neurophotonics 1, 015005. https://doi.org/10.1117/1.NPh.1.1.015005 (2014).

48. Khalid, M. et al. Development of a stand-alone DCS system for monitoring absolute cerebral blood flow. Biomed. Opt. Exp. 10, 4607-4620. https://doi.org/10.1364/boe.10.004607 (2019). 
49. Diop, M., Wright, E., Toronov, V., Lee, T.-Y. \& St. Lawrence, K. Improved light collection and wavelet de-noising enable quantification of cerebral blood flow and oxygen metabolism by a low-cost, off-the-shelf spectrometer. J. Biomed. Opt. 19, 057007. https:// doi.org/10.1117/1.JBO.19.5.057007 (2014).

50. Kewin, M. et al. Evaluation of hyperspectral NIRS for quantitative measurements of tissue oxygen saturation by comparison to time-resolved NIRS. Biomed. Opt. Exp. 10, 4789-4802. https://doi.org/10.1364/BOE.10.004789 (2019).

51. Duncan, A. et al. Optical pathlength measurements on adult head, calf and forearm and the head of the newborn infant using phase resolved optical spectroscopy. Phys. Med. Biol. 40, 295-304 (1995).

52. Cheung, C., Culver, J. P., Takahashi, K., Greenberg, J. H. \& Yodh, A. G. In vivo cerebrovascular measurement combining diffuse near-infrared absorption and correlation spectroscopies. Phys. Med. Biol. https://doi.org/10.1088/0031-9155/46/8/302 (2001).

53. Verdecchia, K., Diop, M., Morrison, L. B., Lee, T.-Y. \& St. Lawrence, K. Assessment of the best flow model to characterize diffuse correlation spectroscopy data acquired directly on the brain. Biomed. Opt. Exp. 6, 4288. https://doi.org/10.1364/boe.6.004288 (2015).

54. Mendelson, A. A. et al. Dynamic tracking of microvascular hemoglobin content for continuous perfusion monitoring in the intensive care unit: Pilot feasibility study. J. Clin. Monit. Comput. https://doi.org/10.1007/s10877-020-00611-x (2020).

55. Highton, D. et al. Monitoring cerebral autoregulation after brain injury: Multimodal assessment of cerebral slow-wave oscillations using near-infrared spectroscopy. Anesth. Analg. 121, 198-205. https://doi.org/10.1213/ANE.0000000000000790 (2015).

56. Grinsted, A., Moore, J. C. \& Jevrejeva, S. Application of the cross wavelet transform and wavelet coherence to geophysical time series. Nonlinear Process. Geophys. 11, 561-566. https://doi.org/10.5194/npg-11-515-2004 (2004).

57. Scholkmann, F., Spichtig, S., Muehlemann, T. \& Wolf, M. How to detect and reduce movement artifacts in near-infrared imaging using moving standard deviation and spline interpolation. Physiol. Meas. https://doi.org/10.1088/0967-3334/31/5/004 (2010).

58. Beausoleil, T. P., Janaillac, M., Barrington, K. J., Lapointe, A. \& Dehaes, M. Cerebral oxygen saturation and peripheral perfusion in the extremely premature infant with intraventricular and/or pulmonary haemorrhage early in life. Sci. Rep. 8, 6511. https://doi. org/10.1038/s41598-018-24836-8 (2018).

59. Mitra, S. et al. Pressure passivity of cerebral mitochondrial metabolism is associated with poor outcome following perinatal hypoxic ischemic brain injury. J. Cereb. Blood Flow Metab. 39, 118-130. https://doi.org/10.1177/0271678X17733639 (2019).

60. Chalak, L. F. et al. Novel wavelet real time analysis of neurovascular coupling in neonatal encephalopathy. Sci. Rep. 7, 45958. https:// doi.org/10.1038/srep45958 (2017).

\section{Acknowledgements}

This study was funded by Canadian Institutes of Health Research (CGP-14171) and National Science and Engineering Research Council (CHRP 478470).

\section{Author contributions}

Conceptualization, K.St.L., M.D., S.B.; methodology, A.R., D.M., L.Y., M.K., L.K.; software, A.R., D.M., M.K; investigation, A.R., M.K., L.K., S.B., S.d.R.; data curation, A.R., M.D., M.K., M.S., L.K., S.B., K.St.L.; writingoriginal draft preparation, A.R., D.M., M.S., K.St.L.; writing-review and editing, A.R., D.M., M.S., S.d.R., V.H., S.B., M.D., K.St.L.; supervision, K.St.L., S.d.R., M.D., S.B., and V.H.; funding acquisition, K.St.L. All authors have read and agreed to the published version of the manuscript.

\section{Funding}

Canadian Institutes of Health Research, CGP-14171, Natural Sciences and Engineering Research Council of Canada, CHRP 478470.

\section{Competing interests}

The authors declare no competing interests.

\section{Additional information}

Supplementary Information The online version contains supplementary material available at https://doi.org/ 10.1038/s41598-021-03830-7.

Correspondence and requests for materials should be addressed to A.R. or K.S.L.

Reprints and permissions information is available at www.nature.com/reprints.

Publisher's note Springer Nature remains neutral with regard to jurisdictional claims in published maps and institutional affiliations.

(c) Open Access This article is licensed under a Creative Commons Attribution 4.0 International License, which permits use, sharing, adaptation, distribution and reproduction in any medium or format, as long as you give appropriate credit to the original author(s) and the source, provide a link to the Creative Commons licence, and indicate if changes were made. The images or other third party material in this article are included in the article's Creative Commons licence, unless indicated otherwise in a credit line to the material. If material is not included in the article's Creative Commons licence and your intended use is not permitted by statutory regulation or exceeds the permitted use, you will need to obtain permission directly from the copyright holder. To view a copy of this licence, visit http://creativecommons.org/licenses/by/4.0/.

(c) The Author(s) 2022 